\title{
Oral immunotherapy for IgE-mediated cow's milk allergy in children: a systematic review and meta-analysis
}

\author{
Lujing Tang ${ }^{1}, \mathrm{Yu} \mathrm{Yu}{ }^{1}$, Xiangyuan $\mathrm{Pu}^{2}$, and Jie $\mathrm{Chen}^{1}$ \\ ${ }^{1}$ Zhejiang University School of Medicine Children's Hospital \\ ${ }^{2}$ Zhejiang University School of Medicine First Affiliated Hospital
}

August 2, 2021

\begin{abstract}
Background: Cow's milk allergy(CMA) is the most common allergy in infants that decreases the quality of life of patients and their families. Standard treatment for CMA is the strict avoidance of milk, new treatment strategies such as oral immunotherapy (OIT) have been sought for patients with CMA . We aimed to assess the clinical efficacy and safety of OIT in the treatment of children with IgE-mediated cow's milk allergy (IMCMA). Methods: We searched all randomized controlled trials (RCTs) in which OIT is used to treat children with IMCMA from 5 international electronic databases. We estimated a pooled relative ratio (RR) for each outcome using a Mantel-Haenzel fixed-effect model if statistical heterogeneity was low. Results: Eleven studies were chosen for meta-analysis, including a total of 469 children (242 OIT, 227 control). 176 patients (72.7\%) in the OIT were desensitized compared to 49 patients in the control group (RR 7.35, 95\% CI 2.82-19.13, $\mathrm{p}<0.0001$ ). The desensitization effect of OIT was particularly significant in children over 3 years old (RR 18.05, 95\% CI 6.48-50.26, p<0.00001). Although adverse effects were common, they usually involved mild reactions, but epinephrine use was more common in the OIT group (RR 7.69, 95\%CI 2.16-27.33, $\mathrm{p}<0.002)$. Conclusion: OIT can lead to desensitization in the majority of individuals with IMCMA, especially in patients over 3-years old. A major problem of OIT is the frequency of adverse events, although most are mild. OIT may be an alternative treatment in the future.
\end{abstract}

\section{Introduction}

Cow's milk allergy (CMA) is defined as a reproducible adverse reaction to cow milk protein mediated by an immunologic mechanism, involving immunoglobulin E ( $\mathrm{IgE}$ )-mediated, non-IgE-mediated, or mixed mechanisms $^{[1]}$. IgE-mediated reactions often occur rapidly, typically beginning within minutes to 2 hours from the time of ingestion ${ }^{[2]}$. Clinical manifestations of IgE-mediated cow's milk allergy (IMCMA) include urticaria, angioedema, rhinitis, conjunctivitis, asthma, oral allergy syndrome, gastrointestinal symptoms, and generalized anaphylaxis. Many children with CMA improve before school age, but in some cases, it persists even into adulthood ${ }^{[3,4]}$. The current standard treatment for CMA is strict avoidance and emergency treatment of severe adverse reactions. However, because milk is ubiquitous in store-bought foods, and inhome and restaurant recipes, it is especially difficult to avoid. On the other hand, it is inevitable of accidental exposure to cow's milk, sometimes it can be life-threatening and has a major impact on quality of life ${ }^{[5]}$. A strict avoidance has negative consequences in patients such as a risk of poor nutrition, increased levels of anxiety, and possible unjustified restrictions to further foods, with an increased immunological risk of non-acquiring tolerance ${ }^{[6]}$. So it needs to find some new treatments, such as oral immunotherapy (OIT). OIT can be proposed at different ages as an effective and safe treatment ${ }^{[1,7]}$.

OIT involves the introduction of a very small amount of cow's milk protein and gradual increases of the dose at predetermined intervals. The global aim is to increase the reactive threshold of allergic patients, and finally enable them to ingest a target quantity of allergen without any reaction throughout the treatment (desensitization). Many studies have showed the efficacy of OIT in desensitization, and some of them in 
sustained unresponsiveness. However, there is an ongoing debate about the safety of OIT $^{[7-9]}$. Because of the increasing interest in this topic and emerging studies, it is important to provide an up-to-date systematic review with ongoing updates. The main objective of this meta-analysis is to assess the clinical efficacy and safety of OIT in children with IMCMA as compared to a placebo treatment or milk avoidance.

\section{Methods}

\section{Criteria for considering studies}

Only randomized controlled trials (RCTs) were considered for inclusion, either blinded or open trial designs. Studies were with no language restrictions. The study population comprised children aged 0-18 years with IMCMA. IMCMA should be confirmed by either: 1) a history of immediate clinical reaction and one of a positive skin-prick test (SPT) or specific IgE to cow's milk protein, or 2) a positive open or double-blind, placebo-controlled food challenge (DBPCFC). The positive reactions include urticaria, angioedema, vomiting, diarrhea, abdominal pain, lightheadedness, and/or syncope. We divided patients into 2 groups: a control or placebo group, which was treated with a milk-avoidance diet or placebo, and an active group, in which children received milk OIT. Milk OIT administered by any protocol and OIT with other adjuvant treatments were included. a subgroup analysis was conducted if possible. Patients with non-IgE-mediated CMA were excluded. Studies of other immunotherapies such as sublingual immunotherapy(SLIT), subcutaneous immunotherapy(SCIT), epicutaneous immunotherapy(EPIT) were all excluded.

\section{Outcome measures}

\section{Primary outcomes}

The primary outcome was the efficacy of OIT: the ability to ingest cow's milk without side effects while on therapy or continued daily ingestion.

\section{Secondary outcomes}

1. Ability to ingest a partial serving of cow's milk without adverse reactions (the dose varies according to the different definitions in each study).

2. Side effects during OIT.

3. Change in skin-prick test(SPT) size, specific IgE level, specific IgG4 level Data was analyzed on an intention-to-treat (ITT) basis whenever possible.

4. Subgroup of the effect of adjuvant treatments such as OIT with baked milk or omalizumab was analyzed if possible.

\section{Search methods}

\section{Electronic searches}

We performed a systematic search with no language restrictions of the following bibliographic databases: PubMed, Medline, Embase, BIOSIS citation index (BCI), and the Cochrane Library. Search terms included: "oral immunotherapy" or "oral tolerance" or "oral desensitization", "milk allergy" or "milk hypersensitivity". The search was up-to-date as of April 30, 2021. In addition, we reviewed the references of the articles included to identify potentially relevant citations.

\section{Data collection and analysis}

Titles and abstracts of records retrieved were examined by one reviewer and irrelevant records excluded. We extracted datas about trial characteristics (setting, MOIT regimen, and eligibility criteria), methodological quality, participants and outcomes of interest. Subsequently, two reviewers evaluated full-text records of all potentially eligible studies based on eligibility criteria and filtered out studies for this meta analysis. Disagreements between reviewers were resolved with discussion. 
We assessed the risk of bias of the included studies based on the criteria established by the Cochrane Handbook for Systematic Reviews of Interventions ${ }^{[10]}$. In the meta-analysis of RCTs, dichotomous outcomes were expressed as a relative ratio(RR) with $95 \%$ confidence intervals (CI). Datas were analyzed on an intention-to-treat basis whenever possible. All analyses were performed by Review Manager Version 5.3. We planned to perform subgroup analysis according to the patients' age (three years and older). Sensitivity analyses were conducted to determine the influence of the studies with a high risk of bias on the meta-analysis.

\section{Assessment of heterogeneity and reporting biases}

We assumed that there would be clinical heterogeneity in the studies, including different ages of the study population and differences in immunotherapy protocol. We assessed heterogeneity between studies using the $\mathrm{I}^{2}$ test with a value greater than $50 \%$ representing substantial heterogeneity. We estimated a pooled RR using a Mantel-Haenzel fixed-effect model if $\mathrm{I}^{2}$ test [?] $50 \%$ or a random-effect model if $\mathrm{I}^{2}$ test $>50 \%$. Funnel plot was used to assess potential publication bias.

\section{Results}

\section{Included studies}

Our electronic searches resulted in 2741 records, after removing duplications, screening titles and abstracts, remained 274 records were screened again for eligibility by two reviewers independently. Discrepancies were resolved through discussion. After applying the inclusion and exclusion criteria, we selected 11 studies $^{[11-21]}$ for this meta-analysis (Figure 1). The characteristics of the included studies are summarized in Table 1.

These 11 studies were published between 2007 and 2021, a total of 469 children (242 OIT, 227 control) were included, of which 234 patients (126 OIT, 108 controls) were older than 3 years old, and a subgroup analysis was conducted for these patients. IMCMA was confirmed by a DBPCFC in eight of the studies ${ }^{[12-18,21]}$, and by a simple-blind placebo-controlled food challenge in two studies ${ }^{[11,20]}$. But in the study of Esmaeilzadeh et al ${ }^{[19]}$, IMCMA was diagnosed by a history of immediate onset of symptoms after ingesting cow's milk and positive SPT and/or IgE antibodies to cow milk. Eight studies used continued elimination diet as a control $^{[11,12,15,17-21]}$, whereas the other two studies used a placebo control ${ }^{[13,16]}$, and Pajno et al used soy milk as a control. Most of the included studies used raw cow milk for OIT, but Esmaeilzadeh et al,used baked milk for OIT and Takahashi et al combined OIT with OMB as the treatment group. The efficacy of desensitization was evaluated by identifying the maximum tolerated dose of milk in the individual studies, as follows: $240 \mathrm{ml}^{[19]} ; 200 \mathrm{~mL}^{[11,14-18,20]} ; 150 \mathrm{~mL}^{[12]} ; 100 \mathrm{ml}^{[21]}$ and $500 \mathrm{mg}^{[13]}$. Four studies included patients younger than three years old $\left.{ }^{[11}, 15,17-18\right]$, and two studies included only children with a history of severe anaphylaxis to milk ${ }^{[12,18]}$, while other three studies excluded such patients ${ }^{[13,15,21]}$, and the rest studies included patients with any degree of reaction. The OIT protocol was different in each study, most of them involved a build-up phase in an institution (hospital, clinic, or research center) followed by periodic up-dosing (either in a clinic or at home) and maintenance at home, but Salmivesi et al conducted OIT trial in the outpatient clinic, and Takahashi et al didn't illustrate this point.

\section{Assessment of Quality}

Figure $2 \mathrm{~A}, 2 \mathrm{~B}$ presents the assessment of the risk of bias of the 11 included studies. There was an appreciable publication bias between included studies by using funnel plots (Figure 2C).

\section{Effect of Interventions}

\section{Desensitization}

The major objective of our meta-analysis was to determine the efficacy of OIT for IMCMA. All 11 studies described the efficacy of OIT, a total of 469 patients were quantitatively analyzed (242 OIT, 227 control). Our meta-analysis showed that 176 patients $(72.7 \%)$ of the patients receiving OIT were able to completely desensitized compared to 49 (21.6\%) of the control group, with a pooled RR of 7.35 (95\% CI 2.82, 19.13; $\mathrm{p}<0.0001$; Figure 3A). After weeding out patients younger than 3-years-old, there was no heterogeneity between the rest studies. So we did a subgroup analysis for these patients over 3-years-old, the result showed 
that $74(58.7 \%)$ patients in the OIT group achieved desensitization, while no one in the control group, with a pooled RR of 18.05(95\% CI 6.48, 50.26; p $<0.00001$; Figure 3B). Because of obvious publication bias, we also performed a sensitivity analysis to ensure that any included study would not affect the overall results (Table 2). In addition, there were five studies that described the effect of OIT on partial desensitization. The definition of partial desensitization is not the same between studies. The analysis showed that the OIT group had a higher rate of partial desensitization than the control group (RR 9.94, 95\% CI 2.8, 34.37; $\mathrm{p}=0.0003$; Figure 3C).

\section{Adverse events}

Six studies were included for analyzing the serious adverse events of OIT. There were only six patients together who experienced serious adverse events, five from the OIT group and one from the control group, with a pooled RR of 2.2 (95\% CI 0.59, 8.22; $\mathrm{p}=0.24$; Figure $4 \mathrm{~A})$, there is no statistical difference. In addition, there were also six studies describing non-serious adverse events of OIT, $82.1 \%(101 / 123)$ in the OIT group compared to $17.5 \%(20 / 114)$ in the control group, the RR value was 4.21 (95\% CI 2.9, 6.13; $\mathrm{p}<0.00001$; Figure 4B), there is a statistical difference. We also analyzed epinephrine use and treatment discontinuation during OIT. There were respectively six and eight studies included and the RR value was 7.69 (95\% CI 2.16, 27.33; $\mathrm{p}=0.002$; Figure 4C) for epinephrine use and 2.23 (95\% CI 0.93, 5.34; $\mathrm{p}=0.07$; Figure $4 \mathrm{D}$ ) for treatment discontinuation. There is a statistical difference for epinephrine use, but not for treatment discontinuation.

\section{Immunological Changes}

We intended to analyze the immunological changes before and after the intervention, such as the change of cow's milk specific IgE, casein IgE, $\beta$-lactoglobulin IgE, $\alpha$-lactalbumin IgE, IgG4 and SPT size, but because of different expression in each study, some used mean value and some used median value, so it was impossible to do systemic analysis.

\section{Other adjuvant therapies combined with OIT}

We also hoped to analyze the efficacy of other adjuvant therapies combined with OIT, for example, OIT with baked milk or OIT combined with omalizumab (OMB). Among our included studies, there was only one study associated with baked milk and OMB respectively. Esmaeilzadeh and colleagues ${ }^{[19]}$ used baked milk for OIT, 42 patients underwent exposure to baked milk in form of muffin for 6 months, and then to cheese in form of pizza for another 6 months if patients could undergo oral challenge test by baked cheese exposure in form of pizza. By the end of the study, 88.1\% (37/42) of the patients in the case group and $66.7 \%(28 / 42)$ of those in the control group had developed milk tolerance $(\mathrm{p}=0.018)$, and the median wheal size of milk extract skin prick test (SPT) in case group demonstrated a decrease pre- and post-baked product in diet [64.3\% (27/42) VS $26.2 \%(11 / 42), \mathrm{p}=0.033]$. Takahashi and colleagues ${ }^{[18]}{ }_{\text {investigated the efficacy of OIT }}$ with OMB. In the treatment group, the patients accepted OMB from the beginning of the study every 2 to 4 weeks until 24 weeks, then OIT was started after the first 8 weeks of OMB treatment, and was maintained for 32 weeks. At weeks 32, all 10 OMB-OIT treated patients and none of the 6 untreated patients passed DBPCFC $(\mathrm{p}<0.001)$. A significantly decreased SPT diameter was found in the OMB-OIT treated group $(\mathrm{P}<0.05)$.

\section{Discussion}

As we all know, there are more and more studies investigating the utility, effectiveness, and drawbacks of milk OIT in patients with IMCMA. On the other hand, it is also effective on partial desensitization, but according to different studies, the OIT protocol used and the duration time are varied in each study. The effort has been done to improve the usefulness of the technique and establishing protocols for more widespread use.

We conducted this systematic analysis of all RCTs in which OIT was used as a treatment for IMCMA. After comparing with the control group, we found that OIT with cow's milk can be an effective and safe alternative therapy. Although there were three meta-analyses on milk OIT published previously ${ }^{[22-24]}$, but the most recently published literature was seven years ago, so it is necessary to do an update meta-analysis 
that include the latest studies. In this meta-analysis, we included five new studies published after the year 2014. According to our analysis, we get conclusions on the efficacy of OIT similar to the previous Metaanalysis, but the number of patients in our meta-analysis is larger than the former three meta-analyses. Furthermore, we also analyzed the effect of OIT on partial desensitization, as well as Yeung and Brozek did. There was no study describing partial desensitization among the new five studies, so we included the same studies with Yeung, but we performed an intention-to-treat analysis. Although there was heterogeneity among included studies, it may be associated with patients'age, small patients' number, different protcol and so on, so we did a subgroup analysis and sensitivity analysis, and obtained similar conclusions.In 2011, Fisher et al ${ }^{[25]}$ published the first meta-analysis on the efficacy of OIT on food allergy and the authors concluded that OIT cannot be recommended in daily practice for desensitization in children with IgE-mediated food allergy. However, they evaluated the effect of OIT in all food allergies not just CMA. In 2017, Nurmatov et $\mathrm{al}^{[26]}$ conducted a meta-analysis on allergen immunotherapy for IgE-mediated food allergy. In this article, the authors involved not only RCTs but also non-RCTs, and food allergies contained CMA, hen's egg allergy, peanut allergy, and so on. The allergen immunotherapy used in this study was OIT, SLIT and EPIT. We didn't include the other six randomized trials ${ }^{[27-32]}$. Staden et al ${ }^{[27]}$ described the total efficacy of OIT on CMA or hen's egg allergy together. Keet and colleagues ${ }^{[28]}$ conducted a randomized trial to explore the safety and efficacy of OIT and SLIT for CMA. Wood and colleagues ${ }^{[29]}$ examined the effect of OMB with OIT comparing with OIT alone. Flore et $\mathrm{al}^{[30]}$ prospectively evaluated the efficacy and the safety of two OIT protocols in a cohort of children with persistent IMCMA: a cluster schedule starting immediately with raw milk versus a slow-progression schedule starting with baked milk and then less and less heated milk over time. In this context, the authors didn't use placebo or milk avoidance as a control group, and they described the efficacy of the two treatment groups together. Chisato and colleagues ${ }^{[31]}$ conducted a trial to evaluate the efficacy and safety of OIT with partially hydrolyzed cow's milk protein-based formula (pHF) in CMA, they compared pHF-pHF to extensively hydrolyzed cow's milk protein-based formula (eHF)-pHF. Nagakura et al ${ }^{[32]}$ compared OIT with heated milk to OIT with unheated milk, the grouping of the latter two contexts was different from our study.

Adverse events during OIT are common, whereas most are mild-moderate and easily managed. Our analysis showed that there were only six patients with serious adverse events, and none was life-threatening. Comparing to the control group, the OIT group had an increased rate of epinephrine use and treatment discontinuation, but there were only 19 patients (17\%) needing epinephrine use. The indications for epinephrine use in each study were not the same, especially in the studies which including patients with a history of life-threatening anaphylaxis, patients were asked to use epinephrine as soon as possible if there were any symptoms during OIT. We planned to analyze OIT on the impact of quality of life, but there were no RCTs. In 2012, Carraro et al ${ }^{[33]}$ conducted a pilot study about the impact of OIT on quality of life (QoL) in children with CMA, the results showed that the QoL in emotional impact, food anxiety, social limitations, and dietary limitations domains were significantly improved after completing OIT and the improvement seemed particularly evident in children over 4 years old. Epstein-Rigbi et al ${ }^{[34]}$ examined changes in QoL of children with food allergy during the up-dosing phase of OIT, the total Food Allergy Quality of Life Questionnaire-Parent Form (FAQLQ-PF) scores improved in 35\%-50\% of patient, but deteriorated in another $25 \%-30 \%$. The same authors did another similar trial ${ }^{[35]}$ on Food Allergy Quality of Life Questionnaire-Children Form (FAQLQ$\mathrm{CF}$ ) scores and FAQLQ-PF scores, they concluded that the total FAQLQ-CF score of children undergoing OIT improved significantly from the start of OIT to end of up-dosing $(\mathrm{p}<0.001)$, a greater improvement was noted in the children who reached a follow-up visit. Parents reported better quality of life (QOL) scores compared to their children at all stages of OIT, but these two articles included milk, egg, and other food allergies.

Desensitization to CM through immunotherapy has been associated with a decrease in CM-sIgE levels and an elevation in sIgG4 levels ${ }^{[30,32,36-37]}$, suggesting that upregulation of allergen-specific IgG4 responses may be an important event in CM-specific immunotherapy. We intended to analyze the immunological changes before and after the intervention, but most of the included studies described IgE values differently, some in mean and some in the median, so it was impossible to do a combined analysis. On the other hand, only four 
studies described IgG4 level changes $\left.{ }^{[13,14,17} 18\right]$, whereas the values were expressed inconsistently.

To decrease the adverse actions of OIT, many adjuvant therapies combined with OIT were studied. Baked milk is likely to be hypoallergenic in part because of changes in the higher-order structure of conformational epitopes. In the retrospective analysis of Gruzelle et al ${ }^{[38]}$, they found that $42.2 \%$ of children allergic to $\mathrm{CM}$ were desensitized after completing a course of baked milk OIT. This introduction seems to be safe and welltolerated in most cases, but there were also $33.3 \%$ of patients had moderate reactions during OIT, with $18 \%$ OIT interruptions. In our included studies, Esmaeilzadeh and colleagues ${ }^{[19]}$ concluded that OIT with baked milk had a higher rate of milk tolerance. However, in the study of Goldberg et al ${ }^{[39]}$, the authors thought that baked milk-reactive subjects are at high risk of adverse allergic reactions throughout OIT. Furthermore, even in the few selected patients who reached the baked milk maintenance dose, only a limited increase in challenge threshold to unbaked milk was achieved. Caution must be exercised, and further studies especially RCTs are warranted before baked-milk OIT is used freely. Omalizumab (OMB) is a humanized monoclonal anti-IgE antibody that binds to the heavy chain constant $\mathrm{CH} 3$ domain of the free IgE molecule and prevents IgE from binding to FceRI effector cells. It was initially approved in 2003 for the treatment of severe allergic asthma in adolescents and adults. There were several studies of OMB with milk OIT $^{[18,29,40-41]}$, including one pilot study, two RCTs (one comparing to milk avoidance, the other comparing to OIT alone), and one case series. OIT with OMB may allow a shorter build-up phase or higher median tolerated dose, but adverse reactions, including the need for epinephrine, still occurred. So it needs more RCTs to examine the efficacy and safety of OMB.

\section{Conclusion}

Our meta-analysis showed that OIT is effective for children of IMCMA, but the adverse events during OIT cannot be ignored, but most of adverse actions are mild to moderate and epinephrine using is not uncommon. Baked milk and OMB combined with OIT may be new ways to reduce complications, but it needs more large sample size RCTs in the future.

Conflict of interest The authors declare no conflict of interests.

\section{Financial support}

There is no financial support for this review.

\section{Acknowledgment}

The authors were fully responsible for contents and editorial decisions for this manuscript.

\section{Impact Statement}

Our meta-analysis included the latest studies on the efficacy and safety of oral immunotherapy using for IgEmediated cow's milk allergy in children, and the analyses showed that oral immunotherapy was effective, especially for patients older than 3-years-old. But the adverse events during oral immunotherapy cannot be ignored, even though most adverse actions are mild to moderate, and epinephrine using is not uncommon.

\section{References}

1. Halken S, Muraro A, de Silva D, et al. EAACI guideline: Preventing the development of food allergy in infants and young children (2020 update). Pediatr Allergy Immunol. 2021 Mar 12:1-16.

2. Knol EF, de Jong NW, Ulfman LH, et al. Management of Cow's Milk Allergy from an Immunological Perspective: What Are the Options.Nutrients.2019,11(11):2734.

3. Sicherer SH, Sampson HA. Food allergy: epidemiology, pathogenesis, diagnosis, and treatment. J Allergy Clin Immunol 2014, 133: 291-307.

4. Savage J, Johns CB. Food allergy: epidemiology and natural history. Immunol Allergy Clin North Am 2015, 35: 45-59.

5. Bingol A, Uygun DFK, Akdemir M, et al. Clinical phenotypes of childhood food allergies based on immune mechanisms: A multicenter study. Allergy Asthma Proc. 2021 May 1, 42(3): e86-e95 
6. Boné Calvo J, Clavero Adell M, Guallar Abadía I, et al. As soon as possible in IgE-cow's milk allergy immunotherapy. Eur J Pediatr. 2021 Jan; 180(1):291-294. .

7. Martorell Calatayud C, Muriel García A, Martorell Aragonés A, et al. Safety and efficacy profile and immunological changes associated with oral immunotherapy for IgE mediated cow's milk allergy in children: systematic review and meta-analysis. J Investig Allergol Clin Immunol.2014, 24(5):298-307.

8. Sabouraud M, Biermé P, Andre-Gomez SA, et al. Oral immunotherapy in food allergies: A practical update for pediatricians. Arch Pediatr. 2021 May; 28(4):319-324.

9. Demir E, Ciğerci Günaydın N, Gülen F, et al. Oral Immunotherapy for Cow's Milk Allergy: Five Years' Experience from a Single Center in Turkey. Balkan Med J. 2020 Oct 23; 37(6):316-323.

10. Julian Higgins, James Thoma, Jacqueline Chandler, et al. Cochrane Handbook for Systematic Reviews of Interventions. Version 6.2, Available from http://www.cochrane-handbook.org/: The Cochrane Collaboration, 2021.

11. Morisset M, Moneret-Vautrin DA, Guenard L, et al. Oral desensitization in children with milk and egg allergies obtains recovery in a significant proportion of cases. A randomized study in 60 children with cow's milk allergy and 90 children with egg allergy. Eur Ann Allergy Clin Immunol. 2007, 39(1): 12-19.

12. Longo G, Barbi E, Berti I, et al. Specific oral tolerance induction in children with very severe cow's milk-induced reactions. J Allergy Clin Immunol.2008, 121(2): 343-347.

13. Skripak JM, Nash SD, Rowley H, et al. A randomized, double-blind, placebo-controlled study of milk oral immunotherapy for cow's milk allergy. J Allergy Clin Immunol. 2008,122(6): 1154-1160.

14. Pajno G, Caminiti L, Ruggeri P, et al. Oral immunotherapy for cow's milk allergy with a weekly up-dosing regimen: a randomized single-blind controlled study. Ann Allergy Asthma Immunol. 2010 Nov; 105(5):376-381.

15. Martorell A, De la Hoz B, Ibanez MD, et al. Oral desensitization as a useful treatment in 2-year-old children with cow's milk allergy Clin Exp Allergy.2011,41(9): 1297-1304.

16. Salmivesi S, Korppi M, Makela M J, et al. Milk oral immunotherapy is effective in school-aged children. Acta Paediatr. 2012, 102(2): 172-176.

17. Lee J. H., Kim W.S, Kim H, et al. Increased cow's milk protein-specific IgG4 levels after oral desensitization in 7- to 12-month-old infants. Ann Allergy Asthma Immunol. 2013,111(6): 523-528.

18. Takahashi M, Soejima K, Taniuchi S, et al. Oral immunotherapy combined with omalizumab for high-risk cow's milk allergy: a randomized controlled trial. Sci Rep.2017, 7(1): 17453.

19. Esmaeilzadeh H, Alyasin S, Haghighat M, et al. The effect of baked milk on accelerating unheated cow's milk tolerance: A control randomized clinical trial. Pediatr Allergy Immunol. 2018, 29(7): 747-753.

20. De Schryver S, Mazer B, Clarke A E, et al. Adverse Events in Oral Immunotherapy for the Desensitization of Cow's Milk Allergy in Children: A Randomized Controlled Trial. J Allergy Clin Immunol. 2019, Pract 7(6): 1912-1919.

21. Maeda M, Imai T, Ishikawa R, et al. Effect of oral immunotherapy in children with milk allergy: The ORIMA study. Allergol Int, 2021, 70(2): 223-228.

22. Brozek JL, Terracciano L, Hsu J, et al. Oral immunotherapy for IgE-mediated cow's milk allergy: a systematic review and meta-analysis. Clin Exp Allergy. 2012, 42:363-374.

23. Yeung JP, Kloda LA, McDevitt J, et al. Oral immunotherapy for milk allergy. Cochrane Database Syst Rev. 2012,11: CD009542. doi:10.1002/14651858. CD009542. pub2.

24. Martorell CC, Muriel GA, Martorell AA, et al. Safety and efficacy profile and immunological changes associated with oral immunotherapy for IgE-mediated cow's milk allergy in children: systematic review and meta-analysis. J Investig Allergol Clin Immunol. 2014; 24(5):298-307.

25. Fisher HR, Du Toit G, Lack G. Specific oral tolerance induction in food allergic children: is oral desensitization more effective than allergen avoidance? Arch Dis Child. 2011,96:259-264

26. Nurmatov U, Dhami S, Arasi S, et al. Allergen immunotherapy for IgE-mediated food allergy: a systematic review and meta-analysis.Allergy.2017,72:1133-1147.

27. Staden U, Rolinck-Werninghaus C, Brewe F, et al. Specific oral tolerance induction in food allergy in 
children: efficacy and clinical patterns of reaction. Allergy. 2007, 62: 1261-1269.

28. Keet CA, Frischmeyer-Guerrerio PA, Thyagarajan A, et al. The safety and efficacy of sublingual and oral immunotherapy for milk allergy. J Allergy Clin Immunol 2012, 129:448-455.

29. Wood RA, Kim JS, Lindblad R, et al. A randomized, double-blind, placebo-controlled study of omalizumab combined with oral immunotherapy for the treatment of cow's milk allergy. J Allergy Clin Immunol. 2016 Apr, 137(4):1103-1110.e11.

30. Flore A, Charlotte K, Wanda G, et al. Is a slow-progression baked-milk protocol of oral immunotherapy always a safe option for children with cow's milk allergy? A randomized controlled trial. Clin Exp Allergy.2017 Nov, 47(11):1491-1496.

31. Chisato Inuo, Kenichi Tanaka, Satoko Suzuki, et al. Oral Immunotherapy Using Partially Hydrolyzed Formula for Cow's Milk Protein Allergy: A Randomized, Controlled Trial. Int Arch Allergy Immunol. 2018, 177(3):259-268.

32. Nagakura K, Sato S, Miura Y, et al. A randomized trial of oral immunotherapy for pediatric cow's milk-induced anaphylaxis: Heated vs unheated milk. Pediatr Allergy Immunol. 2021, 32: 161-169.

33. Carraro S, Frigo A.C, Perin M, et al. Impact of oral immunotherapy on quality of life in children with cow milk allergy: a pilot study. Int J Immunopathol Pharmacol.2012, 25(3):793-798.

34. Epstein-Rigbi N, Goldberg MR, Levy MB, et al. Changes in patient quality of life during oral immunotherapy for food allergy. Allergy. 2017, 72:1883-1890.

35. Epstein-Rigbi N, Goldberg MR, Levy MB, et al. Quality of life of children aged 8-12 years undergoing food allergy oral immunotherapy: Child and parent perspective. Allergy. 2020, 75: 2623-2632.

36. Perezabad L, Reche M, Valbuena T, et al. Oral food desensitization in children with IgE mediated cow's milk allergy: immunological changes underlying desensitization. Allergy Asthma Immunol Res. 2017, 9(1):35-42.

37. Ebrahimi M, Gharagozlou M, Mohebbi A, et al. The Efficacy of Oral Immunotherapy in Patients with Cow's Milk Allergy. Iran J Allergy Asthma Immunol. 2017June, 16(3):183-192.

38. Gruzelle V, Juchet A, Martin-Blondel A, et al. Benefits of baked milk oral immunotherapy in French children with cow's milk allergy. Pediatr Allergy Immunol. 2020, 31:364-370.

39. Goldberg MR, Nachshon L, Appel MY, et al. Efficacy of baked milk oral immunotherapy in baked milk-reactive allergic patients. J Allergy Clin Immunol. 2015,136:1601-1606.

40. Nadeau KC, Schneider LC, Hoyte L, et al. Rapid oral desensitization in combination with omalizumab therapy in patients with cow's milk allergy. J Allergy Clin Immunol. 2011,127: 1622-1624.

41. Martorell-Calatayud C, Michavila-Go' mez A, Martorell-Aragone's A, et al. Anti IgE-assisted desensitization to egg and cow's milk in patients' refractory to conventional oral immunotherapy. Pediatr Allergy Immunol. 2016, 27:544-546.Figure LegendsFigure 1. Results from searching for studies for inclusion in the review.

Figure 2. (A) The summary of risk of bias; (B) Each risk of bias item for each included study;

(C) Funnel plot of studies.

Figure 3. Efficacy of OIT (A: Desensitization; B: subgroup analysis of desensitization in patients older than 3-years-old; C: partial desensitization).

Figure 4. Adverse events of OIT(A: serious adverse events; B: non-serious adverse events; C: epinephrine use; D: treatment discontinuation) .

Author Contributions Lujing Tang: Conceptualization(lead); Data curation(lead); Formal analysis(lead); Methodology(lead);Writing-original draft(lead);Writing-review and editing(lead). Yu Yu: Data curation(equal). Xiangyuan Pu: Writing-review and editing(equal). Jie Chen: Conceptualization(lead); Writing-review and editing(equal); Supervision (lead).

\section{Ethical Approval}

All analyses were based on previously published studies, thus, no ethical approval and patient consent are required. 


\section{Hosted file}

Table 1. Characteristics of the Included Studies .xlsx available at https://authorea.com/ users/428637/articles/532450-oral-immunotherapy-for-ige-mediated-cow-s-milk-allergy-inchildren-a-systematic-review-and-meta-analysis

\section{Hosted file}

Table 2. sensitivity analysis.docx available at https://authorea.com/users/428637/articles/ 532450-oral-immunotherapy-for-ige-mediated-cow-s-milk-allergy-in-children-a-systematicreview-and-meta-analysis 


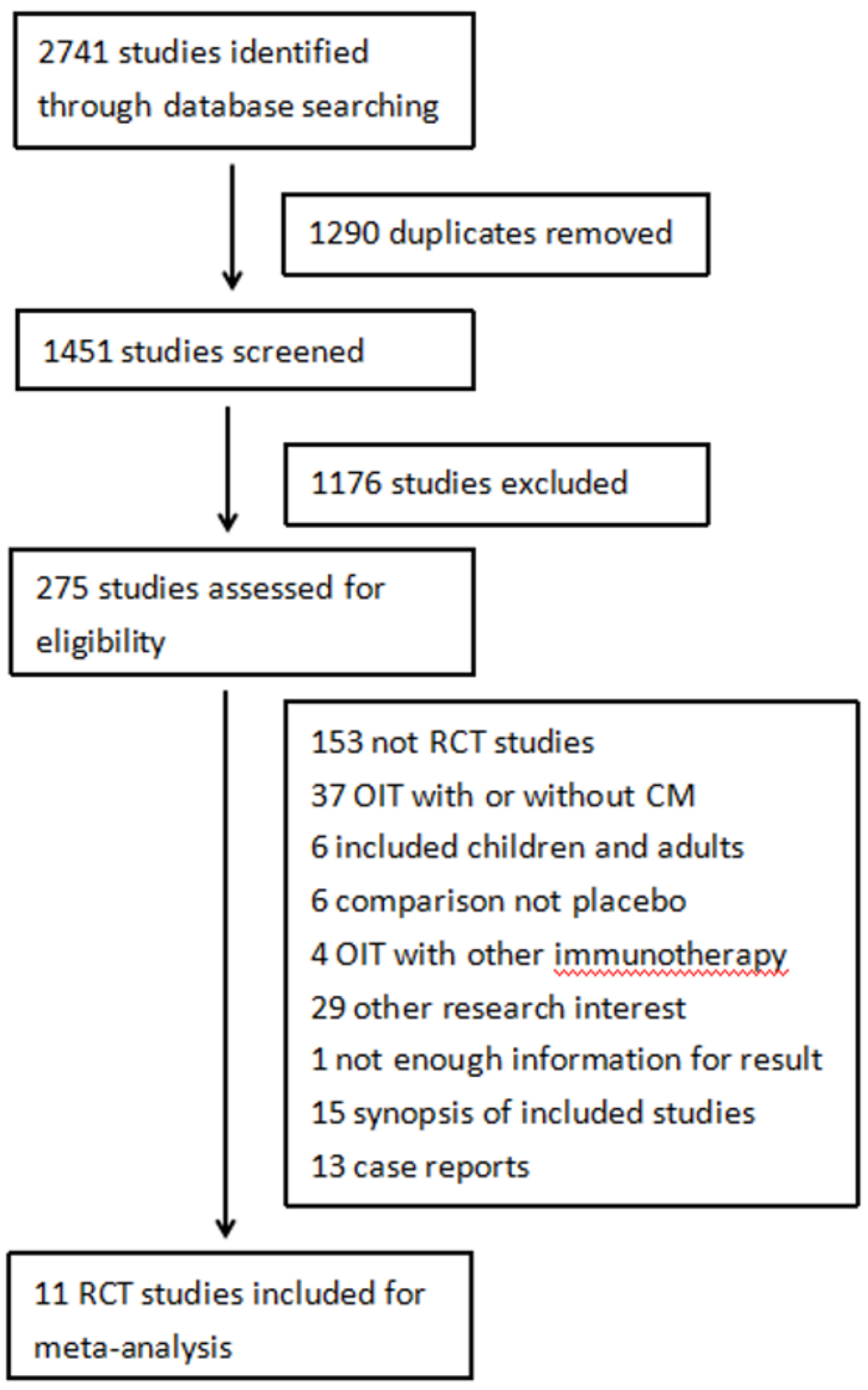




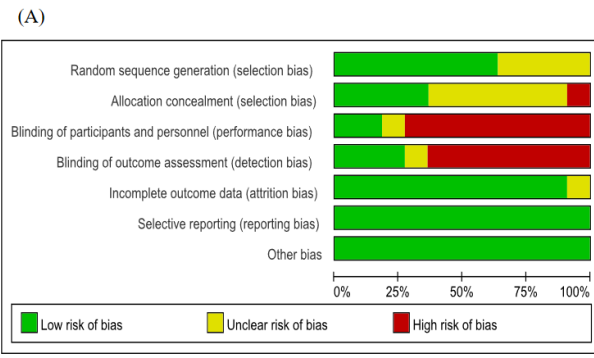

(B)

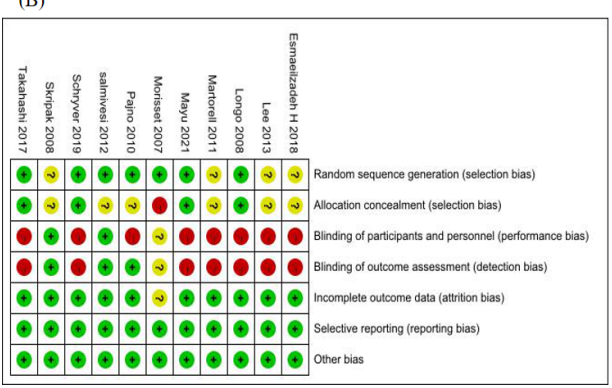

(C)

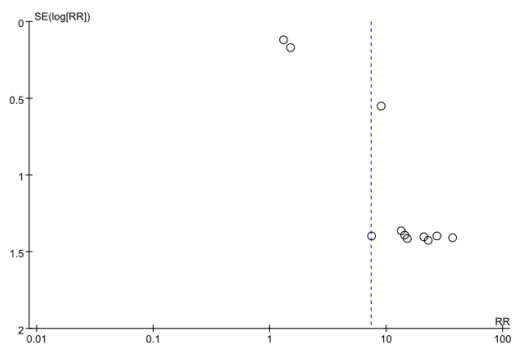

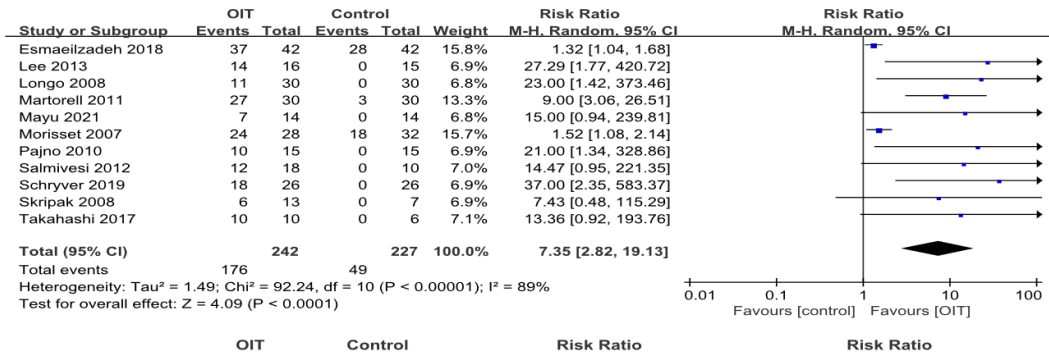

(B) Study or Subgroup OIT Control Risk Ratio Study or Subgroup Events Total Events Total Weight M-H. Fixed. 95\% Cl M-H. Fixed. 95\% Cl Mayu 2021 Pajno 2010 Salmivesi 2012 Schryver 2019
Skripak 2008 Takahashi 2017

Total $(95 \% \mathrm{Cl})$ Total events $\begin{array}{rrlllll}11 & 30 & 0 & 30 & 12.9 \% & 23.00[1.42,373.46] \\ 7 & 14 & 0 & 14 & 12.9 \% & 15.00[0.94,239.81] \\ 10 & 15 & 0 & 15 & 12.9 \% & 21.00[1.34,328.86]\end{array}$ $\begin{array}{lllllll}12 & 18 & 0 & 10 & 16.3 \% & 14.47[0.95,221.35] \\ 18 & 26 & 0 & 26 & 12.9 \% & 3700[2.35 .583 .37]\end{array}$ $\begin{array}{rrrrrr}18 & 26 & 0 & 26 & 12.9 \% & 37.00[2.35,583.37 \\ 6 & 13 & 0 & 7 & 16.4 \% & 7.43[0.48,115.29]\end{array}$ 126 126 Heterogeneity: $\mathrm{Chi}^{2}=0.79, \mathrm{df}=6(\mathrm{P}=0.99) ; \mathrm{I}^{2}=0 \%$ $108 \quad 100.0 \% \quad 18.05[6.48,50.26]$ Test for overall effect: $Z=5.54(P<0.00001)$

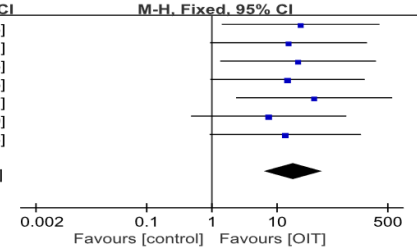
$\begin{array}{cccc}\text { OIT } & \text { Control } & \text { Risk Ratio } \\ \text { (C) Study or Subgroup Events Total Events Total Weight } & \text { M-H. Fixed, } 95 \%\end{array}$ Favours [control] ${ }^{1}$ Favours [OIT] \begin{tabular}{lrrrrrr}
\hline Longo 2008 & 16 & 30 & 0 & 30 & $18.1 \%$ & $33.00[2.07,526.16]$ \\
Martorell 2011 & 1 & 30 & 0 & 30 & $18.1 \%$ & $3.00[0.13 .70 .83]$
\end{tabular} $\begin{array}{lllllll}\text { Martorell } 2011 & 1 & 30 & 0 & 30 & 18.1 \% & 3.00[0.13,70.83\end{array}$ $\begin{array}{lllllll}\text { Pajno } 2010 & 1 & 15 & 0 & 15 & 18.1 \% & 3.00[0.13,68.26] \\ \text { Salmivesi } 2012 & 4 & 18 & 0 & 10 & 22.9 \% & 5.21[0.31,87.93]\end{array}$ $\begin{array}{llrrrrr}\text { Salmivesi } 2012 & 4 & 18 & 0 & 10 & 22.9 \% & 5.21[0.31,87.93] \\ \text { Skripak 2008 } & 6 & 13 & 0 & 7 & 23.0 \% & 7.43[0.48,115.29]\end{array}$

$\begin{array}{lllll}\text { Total }(95 \% \mathrm{Cl}) & 106 & 92 & \mathbf{1 0 0 . 0 \%} & 9.94[2.87,34.37]\end{array}$ Total events

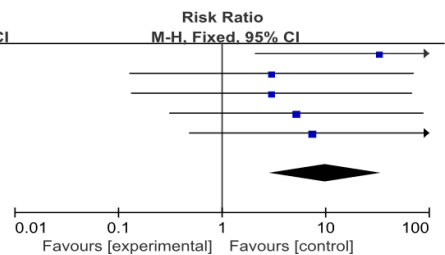


(A)

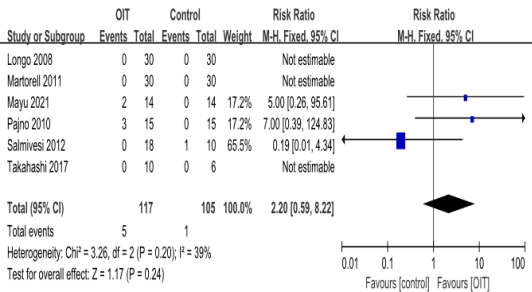

(C)

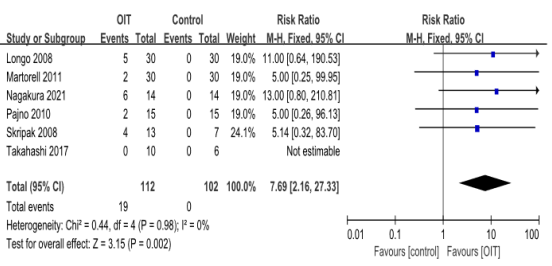

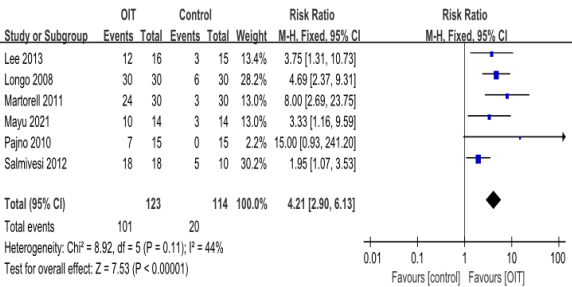

(D) 